\title{
HIGH-RESOLUTION CT (HRCT) CHEST FINDINGS IN PCR POSITIVE PATIENTS OF COVID- 19 AT COMBINED MILITARY HOSPITAL RAWALPINDI
}

\author{
Javed Ahmad Khan, Faryal Asmat*, Fuad Ahmad Siddiqi, Tariq Bashir Tareen, Fayyaz Hassan, Sobia Mehreen, Ayesha Sultan, \\ Amen Ejaz \\ Combined Military Hospital/National University of Medical Sciences (NUMS) Rawalpindi Pakistan, *Fauji Foundation Hospital, Rawalpindi Pakistan
}

\begin{abstract}
Objective: To study High resolution computed tomography chest findings in polymerase chain reaction (PCR) positive patients of COVID-19.

Study Design: Cross sectional study.

Place and Duration of Study: Combined Military Hospital Rawalpindi, from May to Jun 2020.

Methodology: Study included high resolution computed tomography chest of 200 patients admitted to COVID ward who were polymerase chain reaction positive. Patients having previously diagnosed Interstitial lung disease were excluded. Informed consent from patients were taken. Different findings on High resolution computed tomography chest were summarized as percent of total cases. British society of thoracic imaging (BSTI) classification was used to classify high resolution computed tomography chest patterns of COVID-19 pneumonia.

Results: Out of 200 patients included in study, 7 had previous changes on their chest imaging and were excluded. 193 patients (145 males and 48 females) enrolled had a median age of 52 years. One hundred and two patients (52.9\%) showed classical pattern for COVID-19, 81 patients $(41.9 \%)$ had indeterminate changes, 3 patients $(1.6 \%)$ had atypical changes while $7(3.6 \%)$ had normal high resolution computed tomography chest. Four had pleural effusions while 5 had mediastinal lymphadenopathy. One hundred and eight nine $(97.9 \%)$ patients had bilateral lung involvement, $145(75.1 \%)$ had central and peripheral while $48(24.9 \%)$ had peripheral invol-vement only. One hundred and forteen $(59 \%)$ patients had ground glass opacities while $70(36.3 \%)$ had ground glass opacities with consolidation. Nine $(4.7 \%)$ patients presented with consolidation only.

Conclusion: High resolution computed tomography chest and COVID polymerase chain reaction done at the same time gives better diagnostic accuracy and helps in classifying these patients into different categories with early detection and treatment of patients.
\end{abstract}

Keywords: COVID-19, High resolution computed tomography, Polymerase chain reaction.

This is an Open Access article distributed under the terms of the Creative Commons Attribution License (https://creativecommons.org/licenses/by-nc/4.0/), which permits unrestricted use, distribution, and reproduction in any medium, provided the original work is properly cited.

\section{INTRODUCTION}

A no. of patients in China presented to hospital in December 2019 in Wuhan City of Hubei Province. They had fever and lower respiratory tract infection. This was a febrile illness of unknown cause. During course of investigations, a new strain of corona virus was isolated. World Health Organization (WHO) named it as corona virus disease 2019 (COVID-19) ${ }^{1}$. Centers for disease control and WHO developed guidelines for investigation and management of this new disease. One of the major investigations is computed tomography of chest ${ }^{2}$. This modality has been used extensively in the diagnosis of COVID-19 infection which enabled the health professionals to see different patterns of involvement of lung parenchyma ${ }^{3}$. Reverse transcription polymerase chain reaction (RT-PCR) was developed to confirm the diagnosis of COVID-19 infection. This test although being considered as gold standard for COVID-19 is not without certain short comings due

Correspondence: Dr Javed Ahmad Khan, Department of Medicine, Combined Military Hospital Rawalpindi Pakistan

Received: 16 Aug 2020; revised received: 20 Nov 2020; accepted: 24 Nov 2020 to limited availability of kits which in turn lead to less samples being taken. There is also problem of false negative results. It is in these patients where High resolution computed tomography (HRCT) chest performed becomes helpful for prompt diagnosis as it may show changes in lung parenchyma prior to clinical presentation ${ }^{4}$. The commonest change has been ground glass opacities involving different lobes of lungs. This has been reported in literature frequently from very beginning of the disease outbreak ${ }^{5}$. The purpose of our study was to see patterns of HRCT chest findings in PCR positive patients of COVID-19 presenting to a tertiary care hospital.

\section{METHODOLOGY}

The cross-sectional study was undertaken at department of Medicine Combined Military Hospital Rawalpindi with the approval of Institutional review board/ethics committee certificate no. 77/06/20 (26). Informed consent from patients were taken. Patients admitted to our hospital with symptoms suggestive of COVID-19 disease were tested. Nasopharyngeal swabs for COVID-19 PCR were sent. Patients who tested 
positive for COVID-19 were included in the study and their HRCT chest were carried out. Convenience sampling was used and 200 patients who fulfilled the inclusion criteria were included. Seven patients gave past history of respiratory disease including interstitial lung disease, chronic obstructive pulmonary disease and asthma. They also gave history of undergoing imaging in past which showed changes in their lungs. Hence, these patients were excluded. Finally 193 patients were included in study.

HRCT chest were done in supine position on Toshiba Alexian 16 slice CT scan machine. The parameters used included slice thickness of $1 \mathrm{~mm}$, gap of $10.3 \mathrm{~mm}, 120 \mathrm{kV}$ and $112 \mathrm{~mA}$. For reporting, the CT images were displayed with standard lung (window width, 1000-2000 HU; window level - 700-500HU) and mediastinal (window width 300-400 HU; window level 30-50 HU) settings.

Consultant radiologists working in Radiology department of $\mathrm{CMH}$ Rawalpindi (with experience ranging from 5-20 years) reviewed all CT images using a viewing console. The HRCT scans were assessed for the presence of ground glass opacities, consolidation, crazypaving pattern, Centrilobular (tree in bud) tiny nodules, lung cavitations, lymphadenopathy and pleural effusions ${ }^{6}$. Ground glass opacification was defined as increased lung parenchymal attenuation that did not obscure the underlying vessels. Consolidation was defined as opacification with the underlying vessels was obscured ${ }^{7}$. Lymphadenopathy was defined as lymph node size of $>10 \mathrm{~mm}$ in short axis dimension ${ }^{8}$. The outer one-third of lung parenchyma was defined as peripheral, and the rest of the lung was defined as central.

British society of thoracic imaging (BSTI) classification was used to classify HRCT chest patterns of COVID 19 infection. The classical/probable COVID-19 pattern include bilateral peripheral multiple ground glass opacities. They may or may not be accompanied by consolidation or crazy paving. Indeterminate for COVID-19 includes central ground glass opacities with or without consolidations. Non-COVID-19 pattern (atypical) includes consolidation that may be segmental or lobar. It is generally isolated and does not have ground glass opacities. There is no lymphadenopathy, lung cavitations, pleural effusions or tree in bud appearance.

The data was collected and analyzed using SPSS and different findings on HRCT scans were summarized as percent of total cases. Results were presented as tables and comparison done with results at other centre's.

\section{RESULTS}

A total of 200 patients were included in study initially. Seven patients had previous changes on their chest imaging and were excluded. Finally 193 patients were enrolled in the study who had a median age of 52 years (inter quartile range 19-95 years) and comprised 145 males with median age of 50 years (inter quartile range 19-95 years) and 48 females with median age of 57 years (inter quartile range 23-90 years).

Out of 193 patients included in our study, HRCT of 102 patients (52.9\%) showed classical pattern for COVID-19, 81 patients (41.9\%) had indeterminate changes, 3 patients $(1.6 \%)$ had atypical changes while 7 $(3.6 \%)$ had normal HRCT chest. Four of our patients had pleural effusions while 5 had mediastinal lymphadenopathy.

In our study, 189 (97.9\%) patients had bilateral lung involvement. The distribution of involvement in $145(75.1 \%)$ patients was central and peripheral. Forty eight $(24.9 \%)$ patients had peripheral involvement while none had central involvement only. One hundred and fourteen (59\%) of the patients had ground glass opacities while $70(36.3 \%)$ had ground glass opacities with consolidation. Nine $(4.7 \%)$ patients presented with consolidation only.

Since all patients included in our study had positive PCR for COVID 19, they were shifted to another tertiary care hospital which was declared as COVID hospital.

These patients were later shifted to another tertiary care hospital which was declared as Hospital for COVID-19 patients.

Table-I: Classification of High Resolution CT (HRCT) Chest Patterns With Respect to Gender.

\begin{tabular}{l|c|c|c}
\hline $\begin{array}{l}\text { High Resolution } \\
\text { CT Chest Pattern }\end{array}$ & $\begin{array}{c}\text { No. of Cases } \\
\text { n (\%) }\end{array}$ & $\begin{array}{c}\text { Males } \\
\text { n (\%) }\end{array}$ & $\begin{array}{c}\text { Females } \\
\mathbf{n}(\mathbf{\%})\end{array}$ \\
\hline Classical & $102(52.9)$ & $77(39.9)$ & $25(12.9)$ \\
\hline Indeterminate & $81(41.9)$ & $61(31.6)$ & $20(10.4)$ \\
\hline Atypical & $3(1.6)$ & $1(0.5)$ & $2(1.0)$ \\
\hline Normal & $7(3.6)$ & $6(3.1)$ & $1(0.5)$ \\
\hline
\end{tabular}

\section{DISCUSSION}

The study shows that patients who have COVID19 PCR positive are likely to have changes on their HRCT chest. This is important especially in cases where patients have mild symptoms like at the beginning of infection or are PCR negative but have changes on HRCT chest. These patients can be picked up early 
and treatment initiated in the beginning of illness is likely to have better clinical outcomes. was found in $87.1 \%$ patients by Zhao et al15, $60 \%$ of patients by Yoon et al16.

Table-II: Distribution of High Resolution CT (HRCT) Chest Findings According to Age Groups.

\begin{tabular}{|c|c|c|c|c|c|}
\hline High Resolution CT (HRCT) Findings & $\begin{array}{c}20-40 \text { years } \\
\text { n }(\%)\end{array}$ & $\begin{array}{c}\text { 41-50 years } \\
\text { n }(\%)\end{array}$ & $\begin{array}{c}\text { 51-60 years } \\
\text { n ( } \%)\end{array}$ & $\begin{array}{c}\text { 61-70 years } \\
\text { n }(\%)\end{array}$ & $\begin{array}{c}>70 \text { years } \\
\text { n }(\%)\end{array}$ \\
\hline \multicolumn{6}{|l|}{ Lung Involvement } \\
\hline Bilateral & $13(6.7)$ & $22(11.4)$ & $66(34.2)$ & $57(29.5)$ & $31(16)$ \\
\hline Right & - & - & $4(2.1)$ & - & - \\
\hline Left & - & - & - & - & - \\
\hline \multicolumn{6}{|l|}{ Distribution of Involvement } \\
\hline Central & - & - & - & - & - \\
\hline Peripheral & $4(2.1)$ & - & $26(13.5)$ & $9(4.7)$ & $9(4.7)$ \\
\hline Central and Peripheral & $9(4.7)$ & $22(11.4)$ & $44(22.8)$ & $48(24.9)$ & $22(11.4)$ \\
\hline \multicolumn{6}{|l|}{ Density of Lesion } \\
\hline Ground Glass Opacity & $13(6.7)$ & $13(6.7)$ & $35(18.1)$ & $35(18.1)$ & $18(9.3)$ \\
\hline Consolidation and Ground Glass Opacity & - & $9(4.7)$ & $26(13.5)$ & $22(11.4)$ & $13(6.7)$ \\
\hline Consolidation only & - & - & $9(4.7)$ & - & - \\
\hline
\end{tabular}

In our study, most of the patients had bilateral lung involvement $(97.9 \%)$. The distribution of involvement in majority of the patients was central and peripheral $(75.1 \%)$. A portion of patients had peripheral involvement $(24.9 \%)$ while none had central involvement only. More than half of the patients had ground glass opacities (59\%) while others had ground glass opacities with consolidation $(36.3 \%)$. Few patients $(4.7 \%)$ presented with consolidation only. Four patients had pleural effusions while five patients had mediastinal lymphadenopathy.

Qiongjie et al found that the typical early pulmonary CT images of COVID-19 pneumonia were ground glass opacities with or without consolidation, predominantly located peripheral or subpleural location with pulmonary artery dilatation and air bronchograms. However, the early performance of some cases of COVID-19 infection was not typical. About $34.78 \%$ of isolated lesions were located in the central region ${ }^{2}$.

Shi et al found $79 \%$ patients had bilateral lung involvement, $54 \%$ showed peripheral distribution, and $44 \%$ showed diffuse distribution of CT abnormalities. $65 \%$ patients had ground glass opacity while $81 \%$ had ill defined margins, 35\% patients had smooth or irregular interlobular septal thickening, $47 \%$ had air bronchogram and $10 \%$ had crazy paving pattern. Less common CT findings were nodules in $6 \%$ patients, cystic changes $10 \%$, pleural eusion $5 \%$ and lymphadenopathy in $6 \%$ patients $^{9}$. Wang et al reported the mean age of COVID-19 patients as 56 years in their study ${ }^{10}$.

Bilateral distribution was found in $91 \%$ of patients by Caruso et al from Italy ${ }^{11}, 75 \%$ by Bai et al ${ }^{12}, 76 \%$ by Kanne $^{13}$ and $86 \%$ by Song et al ${ }^{14}$ Peripheral distribution
Ground glass opacities (GGO) were reported as $77 \%$, consolidation and GGO as 59\% and consolidation as $55 \%$ by Song et al ${ }^{14}$ Long et al reported GGO as $30.6 \%$, consolidation and GGO as $52.7 \%$ and consolidation as $16.7 \% 17$. The meta-analysis of Zhu et al showed bilateral lung involvement in $73.8 \%$, multi-lobar involvement in $67.3 \%$ and normal CT findings in $8.4 \%$ patients. The lesion density showed ground-glass opacities in $68.1 \%$, air bronchogram sign in $44.7 \%$, crazy-paving pattern in $35.6 \%$ and consolidation in $32 \%$ patients ${ }^{18}$.

Sample size is relatively small and it is a single centre study. Patient's further follow up was not done as these patients were later shifted to another tertiary care hospital which was declared as Hospital for COVID-19 patients. It is recommended that COVID-19 PCR and HRCT chest should be done in all patients presenting with suggestive symptoms of COVID-19.

\section{CONCLUSION}

HRCT chest and COVID PCR done at the same time give better diagnostic accuracy in identifying patients of COVID-19. It also helps in classifying these patients into different categories with early detection and treatment of patients. The predominant findings on HRCT chest were multiple ground glass opacities with or without consolidation with involvement of one or both lungs.

\section{CONFLICT OF INTEREST}

This study has no conflict of interest to be declared by any author.

\section{REFERENCES}

1. Hosseiny M, Kooraki S, Gholamrezanezhad A, Reddy S, Myers L. Radiology perspective of coronavirus disease 2019 (COVID- 
19): lessons from severe acute respiratory syndrome and middle east respiratory syndrome. AJR Am J Roentgenol 2020; 214(5): 1078-82.

2. Hu Q, Guan H, Sun Z, Huang L, Chen C, Ai T, et al. Early CT features and temporal lung changes in COVID-19 pneumonia in Wuhan, China. Eur J Radiol 2020; 128: 109017.

3. Shang Y, Xu C, Jiang F, Huang R, Li Y, Zhou Y, et al. Clinical characteristics and changes of chest $\mathrm{CT}$ features in 307 patients with common COVID-19 pneumonia infected SARS-CoV-2. A multicenter study in Jiangsu, China. Intl J Infect Dis 2020; 96 : 157-62.

4. Dai H, Zhang X, Xia J, Zhang T, Shang Y, Huang R, et al. Highresolution chest $\mathrm{CT}$ features and clinical characteristics of patients infected with COVID-19 in Jiangsu, China. Int J Infec Dis 2020; 95: 106-112.

5. Huang C, Wang Y, Li X, Ren L, Zhao J, Hu Y, et al. Clinical features of patients infected with 2019 novel corona virus in Wuhan, China. Lancet 2020; 6736(20): 1-10.

6. Fu F, Lou J, Xi D, Bai Y, Ma G, Zhao B, et al. Chest computed tomography findings of coronavirus disease 2019 (COVID-19) pneumonia. Eur Radiol 2020; 30(10): 5489-98.

7. Hansell DM, Bankier AA, MacMahon H, McLoud TC, Müller NL, Remy J. Fleischner Society: glossary of terms for thoracic imaging. Radiology 2008; 246(3): 697-722.

8. Chan JF, Yuan S, Kok KH, To KK, Chu H, Yang J, et al. A familial cluster of pneumonia associated with the 2019 novel coronavirus indicating person-to-person transmission: a study of a family cluster. Lancet 2020; 395(10223): 514-23.

9. Shi H, Han X, Jiang N, Cao Y, Alwalid O, Gu J, et al. Radiological findings from 81 patients with COVID-19 pneumonia in Wuhan, China: a descriptive study. Lancet Infect Dis 2020; 20(4):
425-34.

10. Wang D, Hu B, Hu C, Zhu F, Liu X, Zhang J, et al. Clinical Characteristics of 138 hospitalized patients With 2019 Novel Coronavirus-Infected Pneumonia in Wuhan, China. J Am Med Assoc 2020; 323(11): 1061-69.

11. Caruso D, Zerunian M, Polici M, Pucciarelli F, Polidori T, Rucci C, et al. Chest CT Features of COVID-19 in Rome, Italy. Radiol 2020; 296(2): e79-e85.

12. Bai HX, Hsieh B, Xiong Z, Halsey K, Choi JW, Tran TML, et al Performance of Radiologists in Differentiating COVID-19 from Non-COVID-19 Viral Pneumonia at chest CT. Radiol 2020; 296(2): e46-e54.

13. Kanne JP. Chest CT. Findings in 2019 Novel Coronavirus (2019$\mathrm{nCoV}$ ) Infections from Wuhan, China: Key Points for the Radiologist. Radiol 2020; 295(1): 16-17.

14. Song F, Shi N, Shan F, Zhang Z, Shen J, Lu H, et al. Emerging 2019 Novel Coronavirus (2019-nCoV) Pneumonia. Radiology 2020; 295(1): 210-17.

15. Zhao W, Zhong Z, Xie X, Yu Q, Liu J. CT Scans of Patients with 2019 Novel Coronavirus (COVID-19) Pneumonia. Theranostic 2020; 10(10): 4606-13.

16. Yoon SH, Lee KH, Kim JY, Lee YK, Ko H, Kim KH, et al. Chest Radiographic and CT Findings of the 2019 Novel Coronavirus Disease (COVID-19): Analysis of Nine Patients Treated in Korea. Korean J Radiol 2020; 21(4): 494-500.

17. Long C, Xu H, Shen Q, Zhang X, Fan B, Wang C, et al. Diagnosis of the Coronavirus disease (COVID-19): rRT-PCR or CT?. Eur J Radiol 2020; 126: 108961.

18. Zhu J, Zhong Z, Li H, Ji P, Pang J, Li B, et al. CT imaging features of 4121 patients with COVID-19: A meta-analysis. J Med Virol 2020; 92(7): 891-902. 